\title{
Look behind you! The past can contribute uniquely to the future
}

\author{
Elizabeth Griffin ${ }^{1, \star}$ \\ ${ }^{1}$ Dominion Astrophysical Observatory, 5071 West Saanich Road, Victoria, BC, V9E 2E7, Canada
}

\begin{abstract}
The surveys which are the chief foci of this meeting are almost exclusively concerned with recent and upcoming electronic surveys. But the practice of surveying the night sky is not new; some of the first coordinated observatory activities involved surveys, of various hues and sizes, and for many decades the IAU paid tribute to that activity by supporting a special Commission for it. The wealth of past surveys is described briefly. Though now regarded as limited in quality, early surveys carry date-stamps which enable their contents to add usefully, sometimes critically, to modern science.
\end{abstract}

\section{The Future}

Science progresses forwards and upwards. New information creates new pathways and ladders. New knowledge adds another layer on the summit of the pyramid already established by generations of effort and achievement. Like the arrow of time, evolution in our science is inexorably onwards to the Future, and to new things. The Future is the target; the Past was but a facilitator, its parameters superseded, its restrictions overcome, its horizons expanded. Virtually all the contributions reported in these Proceedings demonstrate fascinating individual or collective ability to put ingenuity into practice. Doing so at multiple wavelengths simultaneously can reveal new types of object that had escaped notice through the exercise of sometimes inflexible conventional methods. Moreover, what is freshly learned is not forgotten; the data (the observations) can be mined, the information in whole data-sets can be federated, and new evidence of variability, better-quantified characteristics, and more complete samples, can be analysed, communicated and published. Generations of researchers yet to graduate will work still further on the knowledge which the present ones leave behind them today a never ending search for the wisdom towards which all the effort is striving. Yet despite so many cogent reasons to push onwards and upwards, there will always be some situations in which the Past can also contribute uniquely to modern-day studies, contributions that can be critical when resolving an ambiguity, or (as illustrated in Fig. 1) extending a base-line significantly.

\section{The Past}

Astronomical surveys are certainly not new in concept. As with any pursuit in which a vast uncharted terrain presents itself, an obvious way to begin is to map it - to get a working appraisal of its population, range and scope, and to record that for re-use and for posterity. So with astronomy. The first surveys recorded on physical media (i.e., could be shared) commenced as soon as the resources -

${ }^{\star}$ elizabeth.griffin@nrc-cnrc.gc.ca 
manpower, equipment and detectors - were assembled. Scarcely had the process of photography been commercialized than the Astrographic Catalogue and its deeper-reaching cousin, the Carte du Cielastronomy's first truly international ventures - were conceived in 1887 to record the whole sky, an international project of such grand proportions that it absorbed and nearly exhausted the astronomical resources of some countries, particularly France, for a substantial time. Although the project was never fully completed (the demands eventually exceeded the committed resources), the original observations still exist, and when fully digitized and aligned with modern observations they will enable the recognition of changes in position or magnitude which can be hard to detect when the temporal base-line is very short.

It was in fact the positive experiences and the communications networks set up through the Carte $d u$ Ciel that led to the creation of the IAU in 1919. The importance of the roles that surveys play in astronomical research was fully acknowledged when one of the first of the IAU Commissions to be set up was that for the Carte $\mathrm{du}$ Ciel; it retained its identity as a separate Commission until 2006. Another early Commission (Instrumentation) later diversified and sub-divided as technology burgeoned, and in 1991 it updated its Working Group for Astronomical Photography to the Wide-Field Imaging WG, expressly encompassing Sky Surveys and Patrols and begetting the Wide-Field Plates Database ${ }^{1}$, astronomy's virtual catalogue of photographic wide-field images.

Other surveys with parallel purposes, though smaller in terms of observatory involvement, also started to flourish in the late $19^{\text {th }}$ and early $20^{\text {th }}$ centuries. Probably the best known is the collection of Harvard College Observatory, featuring over half a million images and objective-prism spectra dating back $\sim 130$ years. The on-going programme, $D A S C H^{2}$, to digitize the entire collection is making encouraging headway, and uncovering vast numbers of variable stars. Next in size, the Sonneberg Sky Patrol, initiated in 1926 with relatively modest equipment, delivered some 240,000 plates, led to the detection of over 10,000 variable stars, and laid foundations for $20^{\text {th }}$-century stellar physics. Smaller contemporary surveys, many made in Eastern Europe, provided essential back-up data or filled in gaps in the larger surveys.

Those early observations, while surely not as sophisticated or detailed as the output from modern surveys, or as accessible (unless or until transformed into electronic output), nevertheless offer a baseline extending to a century or beyond, whereas recent surveys cannot by definition encompass more than a handful of years. Observational selection is often a crippling restriction to results from limited surveys, and fascinating new science can be uncovered when its effects are sufficiently reduced. A fine example described by [1] (see Fig. 1) demonstrates how the results of a radial-velocity programme that commenced in 1975 show the actual distribution of binary-star orbital amplitudes and periods to be substantially different from what could previously be deduced from even the most comprehensive catalogue ([2]).

\section{The Present}

There is nothing magical about "the present". It represents neither a particularly advanced nor an especially sophisticated state, since those are relative properties that are closely tied to, and consequently constrained by, the evolution of relevant technology. The present is merely where we now happen to be along the continuum that connects what was with what will be; the Future is some unknown and undefinable state towards which astronomers will always strive but will never know in absolute terms when it is reached. What they can do is to ensure maximal use and application of all the materials to hand at any point, whether recently deposited or in a long-term archive. All of those materials are complementary, unique, and irreproducible.

\footnotetext{
${ }^{1}$ http: //www.wfpdb.org

${ }^{2}$ http://dasch.rc.fas.harvard.edu/project.php
} 

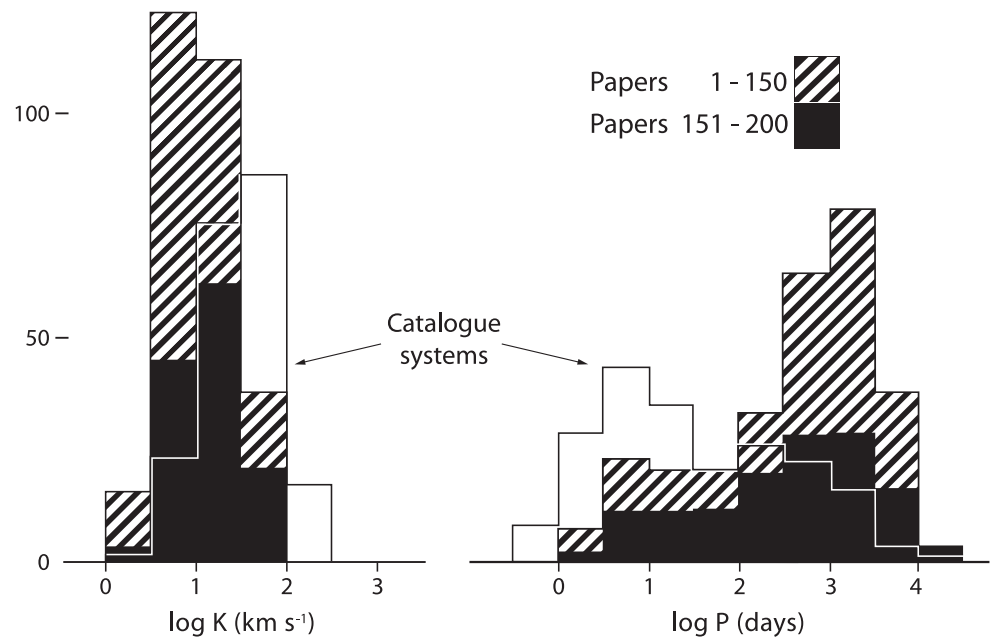

Figure 1. What was believed to be sure knowledge regarding the distribution of amplitudes ( $K$, left) and periods $(P$, right) for tolerably-well determined binary-star orbits listed in the Seventh Catalogue of the Orbital Elements of Spectroscopic Binary Systems [2] (no in-filling) has been substantially altered simply by measuring enough radial velocities over a considerably longer time-span than was customary. Griffin's Papers 1-200 [1] containing the analyses were published at intervals of 2 months. The distributions of $K$ and $P$ are markedly different when the base-line is increased to (a) 25 years (cross-hatching) and (b) 33 years (black). The changes will have had a non-negligible influence on theories of binary-star formation. (Reproduced by kind permission of Observatory Magazine.)

However, where "the present" does offer a once-only chance it is the opportunity to digitize those archived heritage materials before it is too late - before the knowledge about their acquisition and specific properties becomes lost or the data themselves - the photographic plates - destroyed, or discarded in ignorance of their scientific potential. Custom-built digitizing equipment is now available, but will not out-last the plates, and the training and expertise will evaporate even sooner unless passed on to younger members of the community. With those losses will go all the new science whose discovery was the ultimate objective of storing the data for so long.

There is also something important which all astronomers can do to assist, and that is to give support whenever possible to requests for resources to carry out digitizing programmes. Heritage materials are dogged by the stigma that old $\equiv$ old-fashioned, though when they produce evidence of (say) the precursor of a supernova, opinions can change. It should also be pointed out that these heritage materials only need to be digitized once.

Acknowledgments: I am grateful to the Canadian Astronomy Data Centre for a travel grant, and to the conference organizers for additional financial assistance.

\section{References}

[1] Griffin, R. F., Observatory, 128, 448 (2008)

[2] Batten, A. H., Fletcher, J. M., \& Mann, P. J., Seventh Catalogue of the Orbital Elements of Spectroscopic Binary Systems, PDAO, 15, 121, (1978) 